\title{
Tumour tactics
}

\section{Hiroaki Kitano}

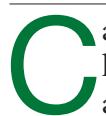
ancer is an extremely complex and heterogeneous disease that exhibits a high level of robustness against a range of therapeutic efforts. Robustness enables a system to maintain functionality in the face of various external and internal perturbations. Robustness is not an accidental property, but follows certain principles. It allows tumours to promote growth and survival in several ways. For example, heterogeneity among tumour cells provides a high level of redundancy, and hence increased chances of survival and growth; these benefits are further enhanced by feedback controls at the cellular level. Viewing cancer as a robust system may provide insight for the development of new drugs and therapies.

In general, carcinomas are heterogeneous and intrinsically redundant populations of cells, generated by transformations including diverse mutations in individual genes, mitotic recombinations, and the loss or gain of entire chromosomes (aneuploidy). This heterogeneous redundancy makes a tumour robust, because survival of any of these proliferating tumour cells allows the cancer to recur. Unfortunately, many anti-tumour drugs are mutagens, and so the diversity of mutations may be increased among surviving cells. In order to control this robustness, an effective therapy needs to reduce or at least avoid increasing this diversity.

This principle has a series of implications. As we are now beginning to understand the possible causes of heterogeneity of tumour cells, the obvious thing to do would be to avoid drugs that may increase heterogeneity by triggering further mutations or anueploidy. The appropriate strategy will depend on the level of heterogeneity and on the drugs available. When the degree of heterogeneity is low, such as in early-stage, non-solid tumours, it would be best to avoid broad-spectrum cytotoxic drugs that may increase heterogeneity, and instead look for one that targets a specific molecule (for example, ABL protein for chronic myelogenous leukaemia). When the level of heterogeneity is high, there is a choice of two strategies. The first is to find a method that actively reduces diversity of mutation among tumour cells — possibly by eliminating all tumour cells except those with a certain mutation or cellular state - then using a drug that specifically targets cells with this property. The alternative would be to guide the malignant cells into dormancy and slow regression — rather than acute and dramatic regressions that impose selective pressures - so that heterogeneity does not increase. However, the drugs used to induce dormancy must be carefully selected, as cytotoxic drugs might cause further mutations, resulting in an increase in heterogeneity despite dormancy, making resistance inevitable.

At the cellular level, feedback control enhances robustness against possible therapeutic efforts. This control, which protects normal cells by making them robust against perturbations, is an obstacle to tumour therapy. Tumour cells counter chemotherapy by overexpressing proteins, such as MDR1, that pump toxic chemicals out of the cell. This simple feedback regulation leads to the acquisition of multidrug resistance. Likewise, the protective role of p53 protein which induces cells to undergo either apoptosis or cell-cycle arrest for DNA repair - is reduced by over-expression of $\mathrm{mdm} 2$, a suppressor of $\mathrm{p} 53$ protein. Thus, modulation of feedback control is a natural strategy for systems-level cancer treatments.

To implement such a strategy, we need to find a method that will systematically control the cell's activities, such as the cell cycle, growth decisions and apoptosis. Computer simulations have shown that a cell cycle that is robust against certain perturbations can be made extremely fragile when specific feedback loops are removed or attenuated, meaning that the cell cycle can be arrested with minimum perturbation. Although this theoretical result has not been experimentally verified, it suggests that robustness can be controlled by carefully selecting modulation targets and the systematic use of multiple drugs. This 'systems drug-discovery' approach aims to control the cell's dynamics, rather than its components, and although only speculative at present, may turn out to be critical for the

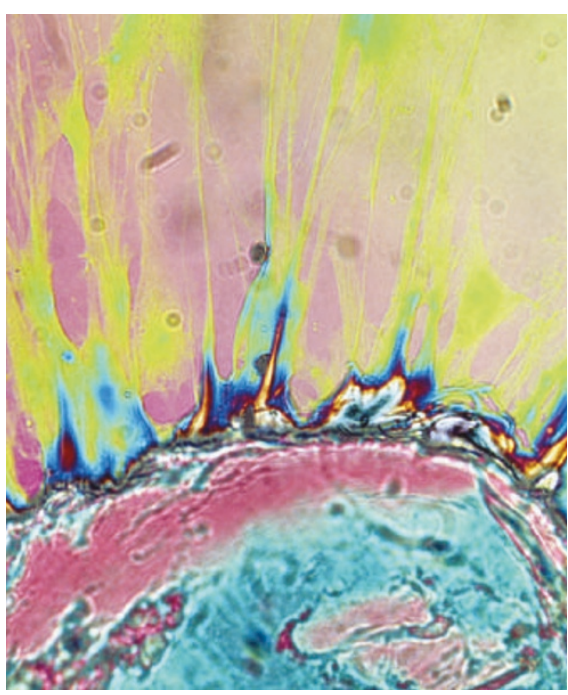

Hunt for fragility: weaknesses in tumour growth dynamics could yield new anti-cancer therapies.

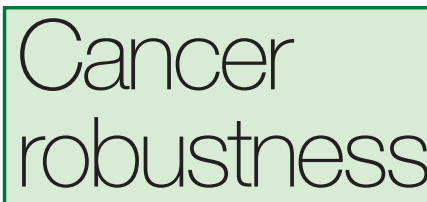

Viewing cancer as a robust system

with potential points of fragility

opens up new strategies for the

development of drugs and

therapies.

development of new cancer therapies.

The ability to predict and control the cellular state may allow exploitation of the trade-off between robustness and fragility. Carlson and Doyle argue in their highly optimized tolerance (HOT) theory that robust complex systems show robustness against common perturbations and extreme fragility against unusual ones. HOT, which takes simple models from statistical physics and adds design or evolution aspects, was originally applied to technologically complex systems such as the Internet, but may be more widely applicable to biological and ecological systems, as demonstrated recently with models of forest fires and abstract lattice evolution. If this theory is relevant to tumours, the existence of extreme robustness implies that there will also be rare points of fragility which, if they can be found, would be a logical target for therapeutic efforts. Although finding such fragility might require a deep understanding of the tumour's regulatory network dynamics, there is potential for new system-level therapies. Ideally, it would be possible to exploit this trade-off to enhance robustness for a specific set of perturbations, so that fragility of the cancer system appears at the point where effective therapeutic agents are readily available.

Considering cancer as a robust system would provide us with a framework for future research strategies. Genetic heterogeneity of tumour cells may be an index of one aspect of robustness that can provide useful information on clinical strategies. An in-depth understanding of the dynamics of cellular robustness could enable us to find methods to control it. Such methods are most likely to be systematic low-dose use of multiple drugs. Future cancer therapies may be judged on their ability to help control the robustness of tumours.

Hiroaki Kitano is at Sony Computer Science Laboratories Inc., 3-14-13 Higashi-gotanda, Shinagwa, Tokyo 141-0022.

\section{FURTHER READING}

Kitano, H. Nature 420, 206-210 (2002).

Morohashi, M. et al. J. Theor. Biol. 216, 19-30 (2002).

Carlson, J. M. \& Doyle, J. Phys. Rev. Lett. 84,

2529-2532 (2000). 